\title{
Myopathy and external pulmonary artery compression caused by sarcoidosis
}

\author{
MM KHAN, DS GILL, B MCCONKEY
}

From the Departments of Medicine and Cardiology, Dudley Road Hospital, Birmingham

Myopathy caused by sarcoidosis, though rare, is now well documented. ${ }^{1}$ Pulmonary artery narrowing from sarcoidosis, however, is extremely rare and only three cases have been previously reported..$^{2-4}$ We describe a patient with sarcoidosis presenting as a rapidly progressive myopathy and pulmonary artery compression. This combination has not been reported before.

\section{Case report}

A 30-year-old Jamaican man was admitted with a month's history of progressive weakness of his arms and difficulty with walking. He was not on any medication. There was no relevant family history. Examination showed mod-

Address for reprint requests: Dr MM Khan, Abdulla Fouad Hospital, PO Box 560, Dammam, Saudi Arabia.

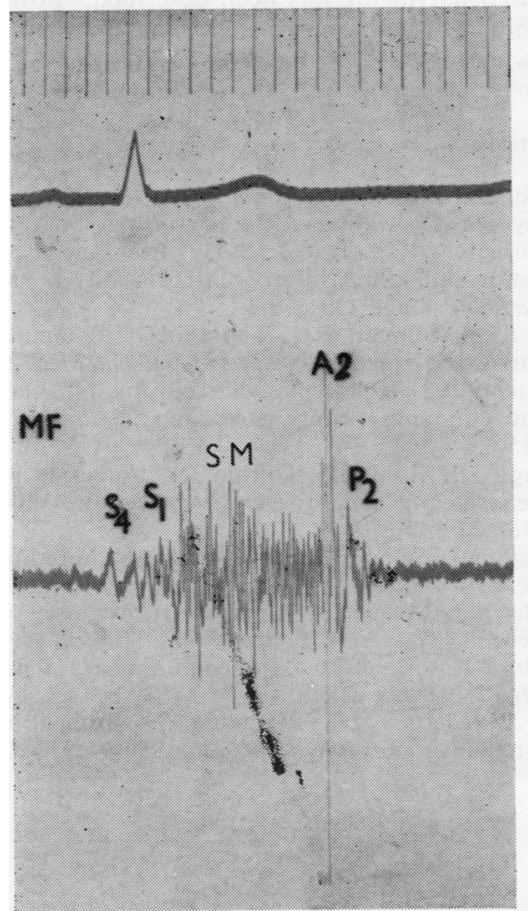

(a) erately severe weakness predominantly involving the proximal limb muscles. There was no wasting, but the muscles were slightly tender. The gait was stiff, waddling and wide-based. The pulse was 64 per minute, regular, BF $115 / 70$. There was no cardiomegaly, or parasternal heave, but there was a harsh systolic murmur in the pulmonary area accompanied by a thrill (fig 1a). The intensity of the murmur varied from day to day. The second heart sound had a variable split. A month earlier he had been admitted to a surgical unit for hypochondrial pain and at that time no heart murmur was noted.

Investigations gave the following results: ESR $44 \mathrm{~mm}$ in the first hour, haemoglobin $12.8 \mathrm{~g} / \mathrm{dl}$, sickling test negative, plasma calcium $2 \cdot 34 \mathrm{mmol} / \mathrm{l}$, urea and electrolytes normal, creatine phosphokinase (CPK) $3383 \mathrm{IU} / 1$, aspartate amino transferase $181 \mathrm{IU} / \mathrm{l}$, no autoantibodies, WR negative, ECG normal. Chest radiography showed a

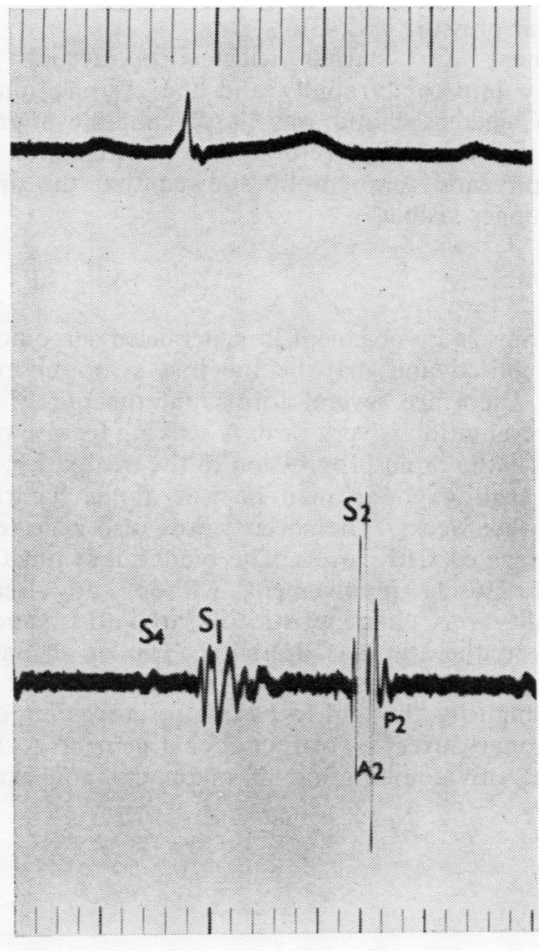

(b)

Fig 1 Mid-frequency phonocardiogram recorded at third left sternal space (a) showing systolic murmur and $(b)$ after steroid treatment murmur no longer present. A2 = aortic component of the second sound, $M F=$ mid-frequency, $P 2=$ pulmonary component of the second heart sound, $S 1=$ first sound, $S 4=$ fourth sound, $S M=$ systolic murmur. 


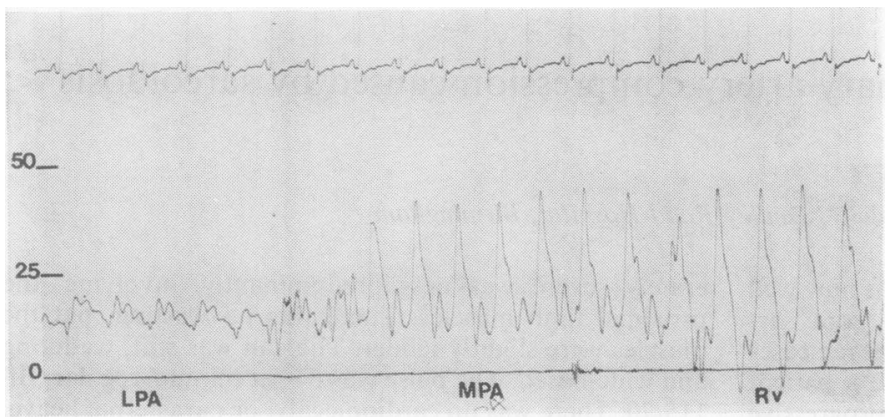

Fig 2 Pull back pressure trace from left pulmonary artery' (LPA) to main pulmonary artery $(M P A)$ to right ventricle $(R V)$.

rounded opacity at the left hilum, which on tomography was approximately $30 \mathrm{~mm}$ in diameter, and close to, but behind, the left main bronchus at $11.5 \mathrm{~cm}$. Echocardiography showed normal mitral and pulmonary valves, normal septal motion, and no evidence of left ventricular outflow obstruction. Muscle biopsy showed noncaseating granulomas, typical of sarcoidosis. Right heart catherisation and pulmonary angiography confirmed the clinical impression of left pulmonary artery compression by what appeared to be an enlarged lymph node. There was a gradient of $23 \mathrm{mmHg}$ on pull back from left pulmonary artery to main pulmonary artery (fig 2 ). Right ventricular pressure was $43 / 6$.

Treatment was started with corticosteroids. The myopathy improved rapidly and the systolic murmur gradually decreased and was hardly audible after two months (fig 1b). CPK returned to normal and chest radiography and tomography showed that the opacity was no longer visible.

\section{Discussion}

Skeletal muscle involvement in generalised sarcoidosis is well recognised and may be the first symptom of the disease. ${ }^{1}$ There are several forms but the purely myopathic presentation is very rare. A striking feature of our patient was the rapid progression of the myopathy; from being a healthy young man he was almost bedridden within a few weeks. The severity was also reflected by grossly elevated CPK levels. The bilateral, symmetrical, proximal muscle involvement without any sensory abnormality was characteristic of sarcoidosis, and the response to therapy was dramatic. The other unusual presenting feature was the loud systolic murmur with variable intensity, thought to be due to narrowing of the left pulmonary artery by glandular enlargement. As far as we know, this combination of myopathy and external pulmonary artery compression caused by sarcoidosis has $\mathrm{W}$ not been described before. Acquired external compression 윽 of the pulmonary artery or its branches from non-vascular causes has rarely been recognised clinically. ${ }^{5-8}$ Respira- $\mathcal{C}$ tory variation of the systolic murmur is considered to be $\mathbb{D}$ highly suggestive of this condition. ${ }^{7}$ Although direct involvement of the small and medium-sized pulmonary vessels by sarcoidosis has been reported, major pulmonary $\stackrel{\mathbb{\Phi}}{-}$ artery narrowing caused by lymphadenopathy is ex- $\vec{P}$ tremely rare. Enlarged lymph nodes caused by sarcoidosis $\infty$ are usually considered to be soft and hence unlikely to :cause pressure upon adjacent bronchus or blood vessels. Bronchial narrowing has been described, ${ }^{9}$ however, and therefore it is not surprising that pulmonary artery narrowing may also be caused.

\section{References}

1 Gardner-Thorpe C. Muscle weakness due to sarcoid myopathy. Neurology 1972;22:917-28.

${ }^{2}$ Faunce HF, Ramsey GC, Sy W. Protracted yet variable major pulmonary artery compression in sarcoidosis. Radiology 1976;119:313-4.

${ }^{3}$ Hietala S, Stinnett RG, Faunce HF. Pulmonary artery narrowing in sarcoidosis. JAMA 1977;237:572-3.

${ }^{4}$ Westcott JL, DeGraff AC. Sarcoidosis, hilar adenopathy and pulmonary artery narrowing. Radiology 1973;108: 585-6.

${ }^{5}$ Gough JH, Gold RG, Gibson RV. Acquired pulmonary stenosis and pulmonary artery compression. Thorax 1967;22:358-67.

${ }^{6}$ Seymour J, Emnuel R, Pattinson N. Acquired pulmonary stenosis. Br Heart $J$ 1968;30:776-85.

7 Littler WA, Meade JB, Hamilton DI. Acquired pulmonary stenosis. Thorax $1970 ; 25: 465-71$.

${ }^{8}$ Dalby AJ, Forman R. Acquired pulmonary stenosis. $S$ Afr $\sigma$ Med J 1978;55:218-20.

${ }^{9} \mathrm{Di}$ Benedetto RJ, Ribaudo C. Bronchopulmonary sarcoidosis. Am Rev Respir Dis 1966;94:952-5. 